\title{
PRODUCTION CORDYLINE FRUTICOSA, L. BY USING TISSUE CULTURE
}

\author{
HANAN M. A. YOUSSEF \\ Ornamental Plants Research and Landscape Gardening Department, Horticulture \\ Research Institute, Agricultural Research Center, Giza, Egypt.
}

(Manuscript received 22 January 2012)

\begin{abstract}
Under in vitro conduction this experiment was carried out at Tissue Culture laboratory at ,Horticulture Research Institute, Agriculture Research Center, during $2009-2010$ year to substitute chemical hormones with natural compound at establishing stage with studying proliferation, rooting and acclimatization of Cordyline fruticosa, explants. The results emphasized that using $20 \%$ Clorox for 20 min for "shoot tips' and 10-20 min, for "one node cutting" with immersion in $70 \%$ ethyl alcohol for five seconds gave the best results $(100 \%)$ survival and free contamination of this explants. Pollen grains collected by hand or bee at $4.00 \mathrm{~g} / \mathrm{l}$ induced significantly increased in callus production, explants development, survival\% , length of shoots, number of formed leaves but not reached to the level of significance in increasing number of formed shoots for both types of explants "shoot tips and one node cutting" . One node cutting failed in rooting by addition the two types of pollen grains to cultured medium. Furthermore the use of $1.0 \mathrm{mg} / \mathrm{l}$ for IBA, NAA or IAA were the best concentrations for callus formation, explants development and survival\% .Using MS medium contained $1.0 \mathrm{mg} / \mathrm{l} \mathrm{NAA}$ and $2.0 \mathrm{mg} / \mathrm{l} \mathrm{BA}$ or KI significantly increased number and length of shoots, BA was better than KI. The shoots of Cordyline fruticosa, successfully rooted when they cultured in MS medium supplemented with $2.0 \mathrm{mg} / \mathrm{I}$ IBA or NAA or IAA. Plantlets after root development formulation of the agricultural media of sand and peat moss at a ratio of $1: 3$ were effective in increasing survival \%,and acclimatization of plant parameters expressed by plant height, shoot thickness and number of leaves.

So, it could be recommend to use pollen grains for substitute chemical hormones but it's not commercial. Cultured explants on MS medium contained $1.0 \mathrm{mg} / \mathrm{l}$.(NAA or IBA or IAA)and $2.0 \mathrm{mg} / \mathrm{IBA}$ for producing the best cordyline production. In addition, using the mixture of sand and peatmoss at a ratio 1-3 for the best acclimatization of Cordyline fruticosa, plant .

Key word: Cordyline fruticosa, $\mathrm{L}$-clorox - pollen grains of date palm-auxin-kinetin-plantlet production.
\end{abstract}

\section{INTRODUCTION}

Cordyline plant (Cordyline fruticosa Family: Liliaceae at last recently Agavaceae)

This is the most popular species, and is grown in immense quantities. The foliage on well-matured plants is of an intense rich crimson marked with lighter shadings. Culturing for the ornamental foliage, plant can be grown as indoor or outdoor attractive plants. It 
tolerates different climatic conditions; i.e. wet and dry weather, high and relatively low temperature.

In vitro propagation can produce large numbers of healthy, homogenous, and identical to their mother plants in short and exact time. Also, reduces losses in plant materials with the lowest expenses which maximize the profitability of the propagation techniques. In the mean time, in vitro propagation encouraged many researchers to study the behavior of the plant and finding out solutions to the problems faced the plants in open climate, i.e. diseases and different stresses. Riordain et. al.. (1983) studied the effect of BA levels on growth, number of shoots and propagules of cordyline produced in vitro. They found that the highest rates of shoots were obtained with the medium supplemented with $0.2 \mathrm{mg} / \mathrm{l} \mathrm{BA}$. Evaldsson (1985) reported that cultured shoots of Cordyline terminalis with various cytokinins. "BA" produced the greatest number of small shoots. The effect of kinetin, zeatin, and isopentenyladenine (2-ip) were similar on these media with few tall shoots were produced than on BA. Shoot length decreased with increasing concentration of BA. For kinetin, zeatin and 2-ip the opposite was true. The number of leaves followed the same pattern. Evaldsson and Welander (1985) found that the largest number of Cordyline terminalis shoots per explant was obtained with $2.0 \mathrm{mg} / \mathrm{l} \mathrm{BA}$ Welander (1988)stated that Murashige and Skoog (MS) medium was suitable for culturing of axillary buds from Cordyline terminalis. Gunidy (1990) stated that NAA was more active than IBA in promoting root development of peach rootstocks.Arafa and Ibrahim (1994) found that the shoots of Cordyline terminalis were increased with B5 supplemented with $3 \mathrm{mg} / \mathrm{l}$ kinetin. Shahid (1999) In vitro propagation of C. terminalis [C. fruticosa] was achieved through induction and proliferation of shoots from stem explants. Callus formation occurred on cut surfaces of shoot apices in 2 to 4 weeks. The largest number of plantlets was produced when callus was maintained by subculturing it at 1-month intervals, 2 to 3 times in MS basal medium. No roots were induced by 2,4-dichlorophenoxyacetic acid (2,4-D), whereas addition of $5.0 \mathrm{mg} \mathrm{NAA} /$ litre improved root growth.6Benzylaminopurine [benzyl adenine] $(0.5 \mathrm{mg} /$ litre) successfully induced adventitious roots within 3 weeks. Ahamed (2009) stated that culturing of shoot tips on modified Murashige and Skoog medium is suitable for direct regeneration. Moreover, using of $2.0 \mathrm{mg} / \mathrm{l} \mathrm{BAP}$ increased proliferation and callus production of Cordyline terminalis.

The ultimate goal of this study is to determine the most suitable balanced medium for in vitro propagation of both Cordyline plants. 


\section{MATERIALS AND METHODS}

\section{- Location and duration}

This investigation was carried out at the Tissue Culture and Germoplasm Conservation Research Laboratory, Horticulture Research Institute, Agriculture Research Center,Giza, Egypt .The experiments were carried out during the period from 2009 to 2010 . The objective of this study was to investigate the most suitable treatments for micropropagation of Cordyline fruticosa.

\section{- Plant material}

The mother plants were grown on Floriculture Research Institute farm. The parts used as explants were Juvenile shoot tip (the terminal plant segment was taken from the apical dome with 3-4 leaf primordia) and one node cuttings, were used in this study.

\section{-Medium consists}

The medium consists of Murashige and Skoog (1962) salts and supplemented with $30 \mathrm{~g} / \mathrm{l}$ sucrose, BA $2.6 \mathrm{mg} / \mathrm{l}$, IAA $1.25 \mathrm{mg} / \mathrm{l}$ and $7.0 \mathrm{~g} / \mathrm{l}$ agar and PH was adjusted at 5.7. The medium was autoclaved at $121^{\circ} \mathrm{C}$ for 20 minutes.

\section{- Experimental treatments}

\section{Surface Sterilization of Explants}

The explants were excised from the mother plants and then washed by soapy water for $5 \mathrm{~min}$ followed by one hour under running tap water. Then they were sterilized by immersion in a clorox (commercial bleach) solution at the rate of 10,20 and $30 \%$ plus 3-5 drops of Tween 20 for 10,15 or $20 \mathrm{~min}$. Then transferred into $70 \%$ ethyl alcohol for five seconds. Finally, they were washed 5 times with sterile distilled water. At the end of the experiments, the collected data included number of survived explants without contamination.

\section{- Culture room condition}

Cultures of Cordyline fruticosa were placed in a growth chamber under $27 \pm 2$ ${ }^{0} \mathrm{C}$ and 16-houres photoperiod provided white fluorescent light (Ahamed, 2009). Sub culturing was done every 4 weeks intervals.

\section{Establishment stage}

For establishment stage, several treatments were initiated from the use of two types of explants. Growth regulators (IAA, IBA and NAA) at 4 levels $(0.0,0.5,1.0$, or $2.0 \mathrm{mg} / \mathrm{l}$ ) were used for establishment stage, comparing with 6 treatments of date palm pollen grains (collected by hand or honey bee in March and April 2009). .In each treatment nine explants in three replicates were cultured for one month. Finally the shoot and callus formation were calculated. 
Table a. General chemical compound of dried pollen grains collected by (hand or bee) according to Tabio,, et. al.., (1988) and Crane (1990).

\begin{tabular}{||c|c|c||}
\hline \multicolumn{1}{|c|}{ Component } & Bee-collected (\%) & Hand-collected (\%) \\
\hline \hline Water (air-dried-pollen) & 11 & 10 \\
\hline \hline Crude protein & 21 & 20 \\
\hline \hline Ash & 3 & 4 \\
\hline \hline Ether extracts (crude fat) & 5 & 5 \\
\hline \hline Reducing sugars & 26 & 3 \\
\hline \hline Non-reducing sugars & 3 & 8 \\
\hline \hline Starch & 3 & 43 \\
\hline \hline Undetermined & 29 & \\
\hline
\end{tabular}

\section{Multiplication stage}

For multiplication stage, the plantlets resulted from establishment stage of Cordyline fruticosa,"shoot tips were used to study the effect of kinetin at different levels i.e.0.0,2.00, 4.00 and $6.00 \mathrm{mg} / \mathrm{l}$ to detect the suitable concentration which induced the highest multiplication during three subculture.

After three subculture, the rate of shoot proliferation was determined for each subculture by recording of the following parameters:

- Number of shoots.

- Shoot length.(cm)

- Number of leaves.

Rooting stage:

In rooting stage, the proliferated shoots were used as explants and cultured on MS medium and treated with IAA,IBA and NAA at 1.00 and $2.00 \mathrm{mg} / \mathrm{l}$ and activated charcool at $1.00 \mathrm{gm} / \mathrm{l}$.

After 30 days on the rooting media the following data were recorded:

- Rooting percentage.

- Number of roots.

- Root length.(cm)

\section{-Acclimatization stag}

Rooted plantlets were pricked out singly into $10 \mathrm{~cm}$ plastic pots filled with 1:1, 2:1 and 3:1 (v/v) peatmoss and sand, respectively. To maintain cultures at high humidity, pots were covered with clear transparent plastic sheets for three weeks. The plastic 
covers were then gradually removed to reduce humidity and to adapt plantlets to greenhouse conditions, after that survival capacity was recorded.

\section{- Experimental design and statistical analysis}

The layout of such factorial experiment was a randomized complete design (Das and Giri,1986) each treatment included five jars was performed containing four explants and each experiment was replicated three times. Data were subjected to analysis of variance by MSTAT-C (1990) Computer statistical analysis program. By using Duncan, Multiple Range Test (1955) to verify the significance level among means of various treatments.

\section{RESULTS AND DISCUSSIONS}

\section{- Effect of different concentrations and periods treatments on surface sterilization explants of Cordyline fruticosa}

Results recorded in Table (1) show that clorox at the rate of $20 \%$ for $15 \mathrm{~min}$ gave the highest value of two explants survival when compared to the other treatments in case of shoot tips. Notably, there were not significant differences between the most concentrations of Clorox at the different times on one node cutting . But, $10 \%$ clorox at 10 or $15 \mathrm{~min}$ and $30 \%$ Clorox at 20 min were 60.0 explants when compared to 80.0 at other treatments on one node cutting. The data indicated that increasing the time of immersed of the explants decreased the survival percentage at the high concentrations of clorox (30\%) while the best concentration $(20 \%)$ increased the free contaminated explants (at $15 \mathrm{~min}$ ) on shoot tips.

The interaction between clorox and time were significant with the highest value of survived explants (83.34), 30\% clorox for 15 min was used.

Table 1. Effect of different concentrations and periods of clorox on surface sterilization\% explants of Cordyline fruticosa.

\begin{tabular}{|c|c|c|c|c|c|c|c|c|}
\hline \multirow{2}{*}{ Time (min) } & \multicolumn{4}{|c|}{ Shoot tips } & \multicolumn{4}{|c|}{ One node cutting } \\
\hline & 10 & 15 & 20 & Mean & 10 & 15 & 20 & Mean \\
\hline Clorox \% & & & & & & & & \\
\hline 10 & $70.00 \mathrm{C}$ & $80.00 \mathrm{~B}$ & $80.00 \mathrm{~B}$ & 72.87B & $60.00 \mathrm{C}$ & $60.00 \mathrm{C}$ & $80.00 \mathrm{~A}$ & 66.67B \\
\hline 20 & $80.00 \mathrm{~B}$ & $80.00 \mathrm{~B}$ & $90.00 \mathrm{~A}$ & 83.34A & $80.00 \mathrm{~A}$ & $80.00 \mathrm{~A}$ & $70.00 \mathrm{~B}$ & 72.87A \\
\hline 30 & $70.00 \mathrm{C}$ & $80.00 \mathrm{~B}$ & $80.00 \mathrm{~B}$ & 72.87B & $70.00 \mathrm{~B}$ & $80.00 \mathrm{~A}$ & $60.00 \mathrm{C}$ & $70.00 \mathrm{~A}$ \\
\hline Mean & $73.34 \mathrm{C}$ & $80.00 \mathrm{~B}$ & 83.34A & & $70.00 \mathrm{~A}$ & $73.34 \mathbf{A}$ & $70.00 \mathrm{~A}$ & \\
\hline
\end{tabular}

Means within column having the same letters are not significantly different according to Duncan's Multiple Range Rest (DMRT) 


\section{- Effect of different concentrations of pollen grains collected by hand or bee on explants establishment of Cordyline fruticosa}

Data in Table (2) demonstrate the effect of pollen grains collected by (hand or bee) on callus production of shoot tips and one node cutting of Cordyline fruticosa,L.,was increased by increasing the concentration level of Pollen grains (hand or bee). Pollen grains (hand or bee) at $4.0 \mathrm{~g} / \mathrm{l}$ was superior than $0.5,1.0,2.0$ or $6.0 \mathrm{~g} / \mathrm{l}$ when compared to explants responded from shoot tips. Starting from $4.0 \mathrm{~g} / \mathrm{l}$ bee pollen grains, plantlet/one node cutting increased to form and showed significant differences when compared to 0.0 and $0.5 \mathrm{~g} / \mathrm{l}$ bee pollen grains $(1.0,2.0$ or $6.0 \mathrm{~g} / \mathrm{l}$ respectively).

Survival percentage of Cordyline fruticosa increased to $80.0 \%$ at $2.0 \mathrm{or} 4.0 \mathrm{~g} / \mathrm{l}$ pollen grains (hand or bee) on two explants used comparing with all treatments.

Table 2. Effect of different concentrations of Pollen grains collected by hand or bee on explants development parameters of Cordyline fruticosa.

\begin{tabular}{|c|c|c|c|c|c|c|c|c|c|c|c|c|}
\hline \multirow{3}{*}{$\begin{array}{l}\text { Parameters } \\
\text { Treatments }\end{array}$} & \multicolumn{4}{|c|}{ Callus production } & \multicolumn{4}{|c|}{ Explants development } & \multicolumn{4}{|c|}{ Survival\% } \\
\hline & \multicolumn{2}{|c|}{ Hand Pollen grains } & \multicolumn{2}{|c|}{$\begin{array}{c}\text { Bee Pollen } \\
\text { grains } \\
\end{array}$} & \multicolumn{2}{|c|}{ Hand Pollen grains } & \multicolumn{2}{|c|}{$\begin{array}{c}\text { Bee Pollen } \\
\text { grains } \\
\end{array}$} & \multicolumn{2}{|c|}{ Hand Pollen grains } & \multicolumn{2}{|c|}{$\begin{array}{c}\text { Bee Pollen } \\
\text { grains } \\
\end{array}$} \\
\hline & S.t. & o.n.c. & S.t. & o.n.c. & S.t. & o.n.c. & S.t. & o.n.c. & S.t. & o.n.c. & S.t. & o.n.c. \\
\hline Control & $0.00 \mathrm{~B}$ & $0.00 \mathrm{~B}$ & $0.00 \mathrm{~B}$ & $0.00 \mathrm{~B}$ & $0.00 \mathrm{D}$ & $0.00 \mathrm{C}$ & $0.00 \mathrm{E}$ & $0.00 \mathrm{D}$ & $0.00 \mathrm{D}$ & $0.00 \mathrm{D}$ & $0.00 \mathrm{D}$ & $0.00 \mathrm{D}$ \\
\hline $0.50 \mathrm{~g} / 1$ & $1.00 \mathrm{AB}$ & $1.00 \mathrm{AB}$ & $1.00 \mathrm{~A}$ & $1.00 \mathrm{~A}$ & $2.00 \mathrm{C}$ & $2.00 \mathrm{~B}$ & $2.00 \mathrm{C}$ & $2.00 \mathrm{C}$ & $60.00 \mathrm{~B}$ & $50.00 \mathrm{~B}$ & $60.00 \mathrm{~B}$ & $50.00 \mathrm{~B}$ \\
\hline $1.00 \mathrm{~g} / \mathrm{I}$ & $1.10 \mathrm{~A}$ & $1.10 \mathrm{~A}$ & $1.00 \mathrm{~A}$ & $1.00 \mathrm{~A}$ & $3.00 \mathrm{~B}$ & $2.50 \mathrm{~A}$ & $2.20 \mathrm{C}$ & $2.40 \mathrm{~B}$ & $60.00 \mathrm{~B}$ & $50.00 \mathrm{~B}$ & $60.00 \mathrm{~B}$ & $50.00 \mathrm{~B}$ \\
\hline $2.00 \mathrm{~g} / \mathrm{I}$ & $1.10 \mathbf{A}$ & $1.10 \mathrm{~A}$ & $1.00 \mathrm{~A}$ & $1.00 \mathrm{~A}$ & $3.25 \mathrm{AB}$ & $2.60 \mathrm{~A}$ & $2.40 \mathrm{~B}$ & $2.40 \mathrm{~B}$ & $80.00 \mathrm{~A}$ & $60.00 \mathrm{~A}$ & $80.00 \mathrm{~A}$ & $60.00 \mathrm{~A}$ \\
\hline $4.00 \mathrm{~g} / 1$ & $1.10 \mathrm{~A}$ & $1.10 \mathrm{~A}$ & $1.00 \mathrm{~A}$ & $1.00 \mathrm{~A}$ & $3.50 \mathrm{~A}$ & $2.70 \mathbf{A}$ & $2.90 \mathrm{~A}$ & $2.60 \mathrm{~A}$ & $80.00 \mathrm{~A}$ & $60.00 \mathrm{~A}$ & $80.00 \mathrm{~A}$ & $60.00 \mathrm{~A}$ \\
\hline $6.00 \mathrm{~g} / \mathrm{I}$ & $1.00 \mathrm{AB}$ & $1.00 \mathrm{AB}$ & $1.00 \mathrm{~A}$ & $1.00 \mathrm{~A}$ & $2.00 \mathrm{C}$ & $2.00 \mathrm{~B}$ & $2.00 \mathrm{D}$ & $2.00 \mathrm{C}$ & $50.00 \mathrm{C}$ & $40.00 \mathrm{C}$ & $50.00 \mathrm{C}$ & $40.00 \mathrm{C}$ \\
\hline Mean & $0.89 \mathbf{A}$ & $0.89 \mathrm{~A}$ & $0.84 \mathbf{A}$ & $0.84 \mathbf{A}$ & $2.29 \mathrm{~A}$ & $1.97 \mathrm{~B}$ & $1.92 \mathbf{A}$ & $1.90 \mathrm{~A}$ & $55.00 \mathrm{~A}$ & 43.34B & $55.00 \mathrm{~A}$ & 43.34 B \\
\hline
\end{tabular}

Means of Pollen grains and pollen bee followed by the same letter within each column are not significantly different from each other at $1 \%$ level.

\section{-Effect of different concentrations of Pollen grains (Hand or bee) on growth and rooting parameters of Cordyline fruticosa:}

The influence of pollen grains (hand or bee) on number of shoots, length of shoots, number of leaves and rooted shoots percentage were tabulated in Table(3)and photo (1)data show significantly variation on responded explants (shoot tips and one node cutting) to Pollen grains (Hand or bee), addition $4.0 \mathrm{~g} / \mathrm{l}$ of Pollen grains (Hand or bee) induced significantly increased of length of shoots and number of leaves comparing with 0.00 (untreated explants), of other concentrations (0.5,1.0,2.0, and $6.0 \mathrm{~g} / \mathrm{l}$ respectively). The number of shoots increased but this increased not significant .One node cutting failed to formed rooting at all treatments. While supplied MS 
medium with 2.0 or $4.0 \mathrm{~g} / \mathrm{l}$ of Pollen grains (Hand or bee) formed $(40.0 \%)$ of rooted in the same time ,other treatments can't formed roots.

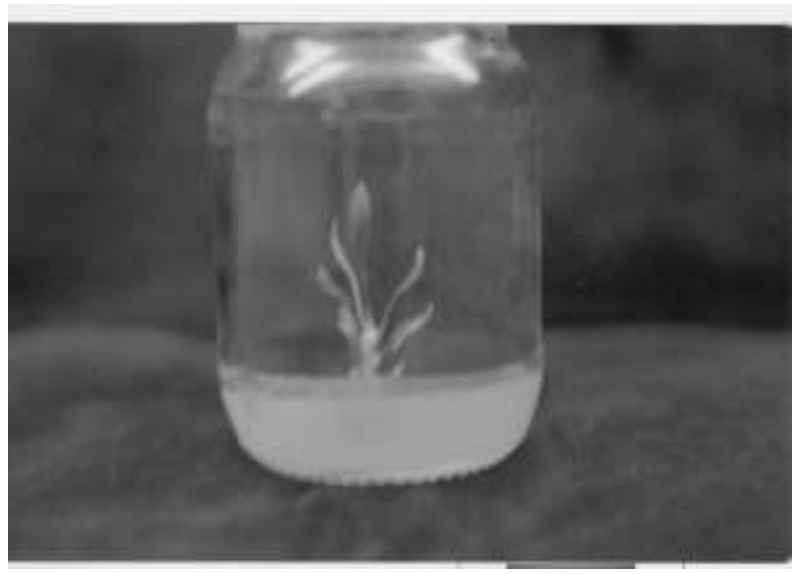

Photo 1. Effect of pollen grains on Cordyline fruticosa, .

Table 3. Effect of different concentrations of Pollen grains collected by hand on growth and rooting parameters of Cordyline fruticosa .

\begin{tabular}{|c|c|c|c|c|c|c|c|c|c|c|c|c|c|c|c|c|}
\hline \multirow[b]{3}{*}{ Treatments } & \multicolumn{4}{|c|}{ Number of shoots } & \multicolumn{4}{|c|}{ Length of shoots } & \multicolumn{4}{|c|}{ Number of leaves } & \multicolumn{4}{|c|}{ Rooted shoots\% } \\
\hline & \multicolumn{2}{|c|}{$\begin{array}{c}\text { Hand Pollen } \\
\text { grains }\end{array}$} & \multicolumn{2}{|c|}{$\begin{array}{c}\text { Bee Pollen } \\
\text { grains }\end{array}$} & \multicolumn{2}{|c|}{$\begin{array}{c}\text { Hand Pollen } \\
\text { grains }\end{array}$} & \multicolumn{2}{|c|}{$\begin{array}{c}\text { Bee Pollen } \\
\text { grains } \\
\end{array}$} & \multicolumn{2}{|c|}{$\begin{array}{c}\text { Hand Pollen } \\
\text { grains }\end{array}$} & \multicolumn{2}{|c|}{$\begin{array}{c}\text { Bee Pollen } \\
\text { grains }\end{array}$} & \multicolumn{2}{|c|}{$\begin{array}{c}\text { Hand Pollen } \\
\text { grains }\end{array}$} & \multicolumn{2}{|c|}{$\begin{array}{c}\text { Bee Pollen } \\
\text { grains } \\
\end{array}$} \\
\hline & S.t. & $\begin{array}{l}\text { o.n. } \\
\text { c. }\end{array}$ & s.t. & $\begin{array}{l}\text { o.n. } \\
\text { c. }\end{array}$ & S.t. & $\begin{array}{l}\text { o.n. } \\
\text { c. }\end{array}$ & s.t. & $\begin{array}{l}\text { o.n. } \\
\text { c. }\end{array}$ & S.t. & $\begin{array}{l}\text { o.n. } \\
\text { c. }\end{array}$ & s.t. & $\begin{array}{l}\text { o.n. } \\
\text { c. }\end{array}$ & S.t. & $\begin{array}{l}\text { o.n. } \\
\text { c. }\end{array}$ & S.t. & $\begin{array}{l}\text { o.n. } \\
\text { c. }\end{array}$ \\
\hline Control & $\begin{array}{c}0.00 \\
\text { B }\end{array}$ & $\begin{array}{c}0.00 \\
\text { B }\end{array}$ & $\begin{array}{c}0.00 \\
\text { B }\end{array}$ & $\begin{array}{c}0.00 \\
\text { B }\end{array}$ & $\begin{array}{c}0.00 \\
\mathrm{E}\end{array}$ & $\begin{array}{c}0.00 \\
\mathbf{E}\end{array}$ & $\begin{array}{c}0.00 \\
\mathbf{E}\end{array}$ & $\begin{array}{c}0.00 \\
\mathbf{E}\end{array}$ & $\begin{array}{c}0.00 \\
\mathbf{F} \\
\end{array}$ & $\begin{array}{c}0.00 \\
\text { D }\end{array}$ & $\begin{array}{c}0.00 \\
\mathbf{C}\end{array}$ & $\begin{array}{c}0.00 \\
\text { B }\end{array}$ & $\begin{array}{c}0.00 \\
\text { B }\end{array}$ & $\begin{array}{c}0.00 \\
\mathbf{A}\end{array}$ & $\begin{array}{c}0.00 \\
\text { B }\end{array}$ & $\begin{array}{c}0.00 \\
\text { A }\end{array}$ \\
\hline $0.50 \mathrm{~g} / 1$ & $\begin{array}{c}1.00 \\
\mathbf{A}\end{array}$ & $\begin{array}{c}1.00 \\
\mathbf{A}\end{array}$ & $\begin{array}{c}1.00 \\
\mathbf{A} \\
\end{array}$ & $\begin{array}{c}1.00 \\
\mathbf{A}\end{array}$ & $\begin{array}{c}2.00 \\
\mathrm{DE}\end{array}$ & $\begin{array}{c}2.00 \\
\text { D }\end{array}$ & $\begin{array}{c}1.10 \\
\text { D }\end{array}$ & $\begin{array}{c}1.20 \\
\mathrm{C} \\
\end{array}$ & $\begin{array}{c}2.00 \\
\mathbf{E} \\
\end{array}$ & $\begin{array}{c}2.00 \\
\mathrm{C}\end{array}$ & $\begin{array}{c}2.00 \\
\text { B } \\
\end{array}$ & $\begin{array}{c}2.00 \\
\mathbf{A}\end{array}$ & $\begin{array}{c}0.00 \\
\text { B } \\
\end{array}$ & $\begin{array}{c}0.00 \\
\mathbf{A}\end{array}$ & $\begin{array}{c}0.00 \\
\text { B }\end{array}$ & $\begin{array}{c}0.00 \\
\mathbf{A} \\
\end{array}$ \\
\hline $1.00 \mathrm{~g} / \mathrm{I}$ & $\begin{array}{c}1.00 \\
\mathbf{A}\end{array}$ & $\begin{array}{c}1.00 \\
\mathbf{A}\end{array}$ & $\begin{array}{c}2.90 \\
\mathrm{C} \\
\end{array}$ & $\begin{array}{c}1.00 \\
\mathbf{A}\end{array}$ & $\begin{array}{c}2.80 \\
\mathrm{C} \\
\end{array}$ & $\begin{array}{r}2.70 \\
\text { BC }\end{array}$ & $\begin{array}{c}1.53 \\
\text { B } \\
\end{array}$ & $\begin{array}{c}1.50 \\
\text { B }\end{array}$ & $\begin{array}{c}2.10 \\
\text { D }\end{array}$ & $\begin{array}{r}2.10 \\
\text { BC }\end{array}$ & $\begin{array}{c}2.00 \\
\text { B }\end{array}$ & $\begin{array}{c}2.00 \\
\mathbf{A}\end{array}$ & $\begin{array}{c}0.00 \\
\text { B }\end{array}$ & $\begin{array}{c}0.00 \\
\mathbf{A}\end{array}$ & $\begin{array}{c}0.00 \\
\text { B }\end{array}$ & $\begin{array}{c}0.00 \\
\mathbf{A} \\
\end{array}$ \\
\hline $2.00 \mathrm{~g} / \mathrm{I}$ & $\begin{array}{c}1.00 \\
\mathbf{A}\end{array}$ & $\begin{array}{c}1.00 \\
\mathbf{A}\end{array}$ & $\begin{array}{c}1.00 \\
\mathbf{A} \\
\end{array}$ & $\begin{array}{c}1.00 \\
\mathbf{A}\end{array}$ & $\begin{array}{c}2.90 \\
\text { B }\end{array}$ & $\begin{array}{c}2.80 \\
\text { B }\end{array}$ & $\begin{array}{c}1.60 \\
\text { B }\end{array}$ & $\begin{array}{c}1.50 \\
\text { B }\end{array}$ & $\begin{array}{c}2.30 \\
\text { B }\end{array}$ & $\begin{array}{l}2.20 \\
\text { A B }\end{array}$ & $\begin{array}{c}2.03 \\
\text { B }\end{array}$ & $\begin{array}{c}2.00 \\
\mathbf{A}\end{array}$ & $\begin{array}{c}40.0 \\
\text { A }\end{array}$ & $\begin{array}{c}0.00 \\
\mathbf{A}\end{array}$ & $\begin{array}{c}0.00 \\
\text { B }\end{array}$ & $\begin{array}{c}0.00 \\
\mathbf{A} \\
\end{array}$ \\
\hline $4.00 \mathrm{~g} / \mathrm{I}$ & $\begin{array}{c}1.00 \\
\mathbf{A}\end{array}$ & $\begin{array}{c}1.00 \\
\mathbf{A}\end{array}$ & $\begin{array}{c}1.00 \\
\mathbf{A}\end{array}$ & $\begin{array}{c}1.00 \\
\mathbf{A}\end{array}$ & $\begin{array}{c}3.02 \\
\mathbf{A}\end{array}$ & $\begin{array}{c}3.00 \\
\mathbf{A}\end{array}$ & $\begin{array}{c}1.90 \\
\mathbf{A}\end{array}$ & $\begin{array}{c}1.60 \\
\mathbf{A}\end{array}$ & $\begin{array}{c}2.60 \\
\mathbf{A}\end{array}$ & $\begin{array}{c}2.30 \\
\mathbf{A}\end{array}$ & $\begin{array}{c}2.15 \\
\mathbf{A}\end{array}$ & $\begin{array}{c}2.00 \\
\mathbf{A}\end{array}$ & $\begin{array}{c}40.0 \\
\text { A }\end{array}$ & $\begin{array}{c}0.00 \\
\mathbf{A}\end{array}$ & $\begin{array}{c}40.0 \\
\mathbf{A}\end{array}$ & $\begin{array}{c}0.00 \\
\mathbf{A}\end{array}$ \\
\hline $6.00 \mathrm{~g} / 1$ & $\begin{array}{c}1.00 \\
\mathbf{A}\end{array}$ & $\begin{array}{c}1.00 \\
\mathbf{A}\end{array}$ & $\begin{array}{c}1.00 \\
\mathbf{A}\end{array}$ & $\begin{array}{c}1.00 \\
\mathbf{A}\end{array}$ & $\begin{array}{c}2.11 \\
\text { D }\end{array}$ & $\begin{array}{c}2.00 \\
\text { D }\end{array}$ & $\begin{array}{c}1.22 \\
\text { C }\end{array}$ & $\begin{array}{c}1.10 \\
\text { D }\end{array}$ & $\begin{array}{c}2.20 \\
\text { C }\end{array}$ & $\begin{array}{l}2.10 \\
\text { BC }\end{array}$ & $\begin{array}{c}2.00 \\
\text { B }\end{array}$ & $\begin{array}{c}2.00 \\
\mathbf{A}\end{array}$ & $\begin{array}{c}0.00 \\
\text { B }\end{array}$ & $\begin{array}{c}0.00 \\
\mathbf{A}\end{array}$ & $\begin{array}{c}0.00 \\
\text { B }\end{array}$ & $\begin{array}{c}0.00 \\
\mathbf{A} \\
\end{array}$ \\
\hline Mean & $\begin{array}{l}0.83 \\
\text { A }\end{array}$ & $\begin{array}{l}0.83 \\
\text { A }\end{array}$ & $\begin{array}{r}0.83 \\
\mathbf{A}\end{array}$ & $\begin{array}{r}0.83 \\
\mathbf{A}\end{array}$ & $\begin{array}{l}2.14 \\
\text { A }\end{array}$ & $\begin{array}{l}2.09 \\
\text { B }\end{array}$ & $\begin{array}{l}1.23 \\
\text { B }\end{array}$ & $\begin{array}{l}1.32 \\
\mathbf{A}\end{array}$ & $\begin{array}{l}1.87 \\
\text { A }\end{array}$ & $\begin{array}{l}1.79 \\
\text { B }\end{array}$ & $\begin{array}{l}1.69 \\
\text { A }\end{array}$ & $\begin{array}{l}1.67 \\
\text { A }\end{array}$ & $\begin{array}{l}13.3 \\
4 \mathbf{A}\end{array}$ & $\begin{array}{l}0.00 \\
\text { B }\end{array}$ & $\begin{array}{l}6.67 \\
\text { A }\end{array}$ & $\begin{array}{l}0.00 \\
\text { B }\end{array}$ \\
\hline
\end{tabular}

Means of Pollen grains and pollen bee followed by the same letter within each column are not significantly different from each other at $1 \%$ level.

\section{- Effect of different concentrations of auxin type on explants development parameters of Cordyline fruticosa}

Data registered in Table (4) demonstrate that NAA, IBA and IAA clearly affected the rooting stage of Cordyline.NAA was superior than IBA and IAA in the 
callus production and survival percentage of shoot tips, in the same time IBA record best results compared with NAA and IAA in explants development.

For NAA, IBA and IAA levels, it was found that $1.0 \mathrm{mg} / \mathrm{l}$ NAA gave the highest callus production explants development and survival percentage of shoot tips and there were significant differences between them and the different concentrations.

One node cutting NAA, IBA and IAA was significant in increasing callus production explants development and survival percentage, which was demonstrated clearly in almost all the different treatments. $1.0 \mathrm{mg} / \mathrm{l}$ was the best for greater in all parameters in three types of auxin compared to control.

\section{- Effect of different ctyokinin types with different concentrations on growth and proliferation parameters of Cordyline fruticosa}

Data in Table (5) clarified the effect of different cytokinin types (KI-BA) with different concentrations on growth and proliferation parameters.

Concerning shoot number, shoot length and number of leaves/shoot increased by increased concentrations of ( $K I$ and $B A$ ).Meanwhile, BA was effective significantly in maximizing shoot number, shoot length and number of leaves/shoot compared with $\mathrm{KI}$..On the other hands, $2.0 \mathrm{mg} / \mathrm{l}$ of $\mathrm{KI} ., 1.0 \mathrm{mg} / \mathrm{l}$ of BA was more suitable for shoot proliferation of Cordyline fruticosa multiplication .

Table 4. Effect of different concentrations of auxin (NAA-IBA-IAA) on explants development parameters of Cordyline fruticosa .

\begin{tabular}{|c|c|c|c|c|c|c|c|c|c|}
\hline \multirow{3}{*}{$\begin{array}{l}\text { Auxin type } \\
\text { Concentration }\end{array}$} & \multicolumn{9}{|c|}{ Shoot tips } \\
\hline & \multicolumn{3}{|c|}{ NAA } & \multicolumn{3}{|c|}{ IBA } & \multicolumn{3}{|c|}{ IAA } \\
\hline & $\begin{array}{c}\text { Callus } \\
\text { p. }\end{array}$ & Exp. D. & Sur. \% & $\begin{array}{c}\text { Callus } \\
\text { p. }\end{array}$ & $\begin{array}{c}\text { Exp. } \\
\text { D. }\end{array}$ & Sur. \% & $\begin{array}{c}\text { Callus } \\
\text { p. }\end{array}$ & $\begin{array}{c}\text { Exp. } \\
\text { D. }\end{array}$ & Sur. $\%$ \\
\hline Control & $0.00 \mathrm{D}$ & $0.00 \mathrm{C}$ & $0.00 \mathrm{D}$ & $0.00 \mathrm{D}$ & $0.00 \mathrm{D}$ & $0.00 \mathrm{D}$ & $0.00 \mathrm{D}$ & $0.00 \mathrm{D}$ & $0.00 \mathrm{D}$ \\
\hline $0.50 \mathrm{mg} / \mathrm{I}$ & $2.01 \mathrm{C}$ & $2.11 \mathrm{~B}$ & $60.00 \mathrm{C}$ & $1.11 \mathrm{~B}$ & $3.25 \mathrm{~B}$ & $70.00 \mathrm{~B}$ & $1.70 \mathrm{C}$ & $1.12 \mathrm{C}$ & $70.00 \mathrm{C}$ \\
\hline $1.00 \mathrm{mg} / \mathrm{l}$ & $3.93 \mathbf{A}$ & $3.02 \mathbf{A}$ & $100.00 \mathbf{A}$ & $1.52 \mathrm{~A}$ & $3.87 \mathrm{~A}$ & $80.00 \mathbf{A}$ & $4.92 \mathbf{A}$ & $2.77 \mathbf{A}$ & $90.00 \mathbf{A}$ \\
\hline $2.00 \mathrm{mg} / 1$ & $3.22 \mathrm{~B}$ & $2.91 \mathbf{A}$ & $80.00 \mathbf{B}$ & $1.00 \mathrm{C}$ & $1.20 \mathrm{C}$ & $60.00 \mathrm{C}$ & $2.45 \mathrm{~B}$ & $2.11 \mathrm{~B}$ & $80.00 \mathrm{~B}$ \\
\hline \multicolumn{10}{|c|}{ One node cutting } \\
\hline Control & $0.00 \mathrm{D}$ & $0.00 \mathrm{D}$ & $0.00 \mathrm{D}$ & $0.00 \mathrm{D}$ & $0.00 \mathrm{D}$ & $0.00 \mathrm{D}$ & $0.00 \mathrm{D}$ & $0.00 \mathrm{D}$ & $0.00 \mathrm{D}$ \\
\hline $0.50 \mathrm{mg} / \mathrm{l}$ & $1.91 \mathrm{C}$ & $1.22 \mathrm{C}$ & $60.00 \mathrm{C}$ & $1.62 \mathrm{~B}$ & $2.22 \mathrm{~B}$ & $70.00 \mathrm{~B}$ & $1.12 \mathrm{C}$ & $1.01 \mathrm{C}$ & $50.00 \mathrm{~B}$ \\
\hline $1.00 \mathrm{mg} / \mathrm{I}$ & $2.63 \mathbf{A}$ & $1.91 \mathbf{A}$ & $80.00 \mathbf{A}$ & $1.91 \mathbf{A}$ & $2.41 \mathrm{~A}$ & $50.00 \mathrm{C}$ & $1.93 \mathbf{A}$ & $1.74 \mathbf{A}$ & $70.00 \mathbf{A}$ \\
\hline $2.00 \mathrm{mg} / \mathrm{I}$ & $2.14 \mathrm{~B}$ & $1.53 \mathrm{~B}$ & $70.00 \mathbf{B}$ & $1.51 \mathrm{C}$ & $1.00 \mathrm{C}$ & $80.00 \mathbf{A}$ & $1.53 \mathrm{~B}$ & $1.23 \mathrm{~B}$ & $50.00 \mathrm{~B}$ \\
\hline
\end{tabular}

Means of Auxin type followed by the same letter within each column are not significantly different from each other at $1 \%$ level. 
Table 5. Effect of different ctyokinin (KI and BA) with different concentrations on growth and Multiplication parameters of Cordyline fruticosa .

\begin{tabular}{|c|c|c|c|c|c|c|}
\hline \multirow[t]{2}{*}{ Treatments } & \multicolumn{2}{|c|}{ Number of shoots } & \multicolumn{2}{|c|}{ Shoot length } & \multicolumn{2}{|c|}{ Number of leaves/ Shoot } \\
\hline & KI & BA & KI & BA & KI & BA \\
\hline Control & 1.55D & $1.40 \mathrm{C}$ & $2.00 \mathrm{~A}$ & $2.80 \mathrm{C}$ & $3.56 \mathrm{~B}$ & $2.37 \mathrm{C}$ \\
\hline $1.00 \mathrm{mg} / \mathrm{I}$ & $2.44 \mathrm{C}$ & 4.53A & $1.89 \mathrm{~B}$ & 4.50A & $3.65 \mathbf{A}$ & 3.40A \\
\hline $2.00 \mathrm{mg} / \mathrm{l}$ & $3.86 \mathbf{A}$ & 4.00B & 1.95 A B & 3.56B & $3.67 \mathbf{A}$ & 3.20B \\
\hline $4.00 \mathrm{mg} / \mathrm{I}$ & 3.45B & 4.00B & $1.90 \mathrm{~B}$ & 2.50D & $3.50 \mathrm{~B}$ & 3.41A \\
\hline Mean & $2.83 \mathrm{~B}$ & $3.49 \mathrm{~A}$ & 1.94 B & $3.34 \mathrm{~A}$ & $3.59 \mathbf{A}$ & $3.09 \mathrm{~B}$ \\
\hline
\end{tabular}

Means of cytokinin type followed by the same letter within each column are not significantly different from each other at $1 \%$ level.

\section{- Effect of different concentrations of auxin on rooting parameters of} Cordyline fruticosa

According to data showed in Table (6) it was generally noticed that addition of $1.0 \mathrm{mg} / \mathrm{L}$ of NAA was significantly improved rooting percentage ,number of root/shootlet and root length parameters as compared with the other treatments. Meanwhile, rooting parameter was statistically increased by using $2.0 \mathrm{mg} / \mathrm{l}$ of IBA or IAA as compared with untreated plantlet (control).The aforementioned results concluded that NAA at $2.0 \mathrm{mg} / \mathrm{l}$ level was effective in rooting .This may be NAA is more active than IAA and IBA in inducing rooting. These results are in general agreement with the findings of Shahid (1999) Cordyline terminalis, found that addition of $5.0 \mathrm{mg} \mathrm{NAA/litre} \mathrm{improved} \mathrm{root} \mathrm{growth.}$

Table 6. Effect of different concentrations of auxin (NAA-IBA-IAA)on rooting parameters of Cordyline fruticosa .

\begin{tabular}{|c|c|c|c|c|c|c|c|c|c|}
\hline \multirow{2}{*}{\begin{tabular}{|l} 
Auxin type \\
Concentration
\end{tabular}} & \multicolumn{3}{|c|}{ NAA } & \multicolumn{3}{|c|}{ IBA } & \multicolumn{3}{|c|}{ IAA } \\
\hline & $\begin{array}{c}\text { Rooting } \\
\text { \% }\end{array}$ & $\begin{array}{c}\text { No.of root/ } \\
\text { shootlet }\end{array}$ & $\begin{array}{l}\text { Root length } \\
\text { (cm) }\end{array}$ & Rooting\% & $\begin{array}{c}\text { No.of } \\
\text { root/ } \\
\text { shootlet }\end{array}$ & $\begin{array}{l}\text { Root } \\
\text { length } \\
(\mathrm{cm})\end{array}$ & $\begin{array}{c}\text { Rooting } \\
\%\end{array}$ & $\begin{array}{c}\text { No.of root/ } \\
\text { shootlet }\end{array}$ & $\begin{array}{l}\text { Root } \\
\text { length } \\
\text { (cm) }\end{array}$ \\
\hline Control & 16.50 D & $1.20 \mathrm{C}$ & $1.22 \mathrm{D}$ & 16.50 D & $1.20 \mathrm{D}$ & $1.22 \mathrm{D}$ & 16.50D & $1.20 \mathrm{D}$ & $1.22 \mathrm{D}$ \\
\hline $0.50 \mathrm{mg} / \mathrm{l}$ & 68.25 B & $2.53 \mathrm{~B}$ & $2.02 \mathrm{C}$ & $35.00 \mathrm{C}$ & $1.80 \mathrm{C}$ & $3.00 \mathrm{C}$ & $26.00 \mathrm{C}$ & $2.23 \mathrm{C}$ & $2.31 \mathrm{C}$ \\
\hline $1.00 \mathrm{mg} / \mathrm{l}$ & $75.23 \mathbf{A}$ & $3.46 \mathbf{A}$ & $3.20 \mathrm{~B}$ & $50.00 \mathrm{~B}$ & $2.66 \mathrm{~B}$ & $3.60 \mathrm{~B}$ & $35.00 \mathrm{~B}$ & $2.41 \mathrm{~B}$ & $4.00 \mathbf{A}$ \\
\hline $2.00 \mathrm{mg} / \mathrm{I}$ & $60.44 \mathrm{C}$ & $3.63 \mathbf{A}$ & $4.12 \mathbf{A}$ & $60.00 \mathbf{A}$ & $3.33 \mathbf{A}$ & $4.00 \mathbf{A}$ & $48.00 \mathrm{~A}$ & $3.42 \mathbf{A}$ & $4.02 \mathbf{A}$ \\
\hline
\end{tabular}

Means of Auxin type followed by the same letter within each column are not significantly different from each other at $1 \%$ level. 


\section{- Effect of soil media on plant growth (acclimazation)}

Data tabulated in Table (7) indicate that using of combination of peatmoss and sand at the ratio of $3: 1$ produced the highest survival percentage $(90 \%)$, plant height (12.99), shoot thickness (1.10), and number of leaves/shootlet (9.67), compared with the other agricultural media either alone or in combinations. However, the worst effect was shown when sand was used alone. These results are in accordance with the findings of Youssef (2003) Yucca elephantipes "Medicus" and Philodendron scandens $\mathrm{L}$.

Table 7. Effect of different agricultural media (Sand and Peatmoss)on growth and rooting parameters of Cordyline fruticosa .

\begin{tabular}{|c|c|c|c|c|}
\hline \multirow{2}{*}{ Parameters } & \multirow{2}{*}{$\begin{array}{c}\text { Survival } \\
\text { Agricultural media }\end{array}$} & Plant height (cm) & No. of leaves & Shoot thickness (cm) \\
\hline Sand & 0.00 & $0.00 \mathrm{E}$ & $0.00 \mathrm{E}$ & $0.00 \mathrm{D}$ \\
\hline Peatmass & 15.00 & $3.55 \mathrm{D}$ & $3.56 \mathrm{D}$ & $0.50 \mathrm{C}$ \\
\hline Peat :Sand 1:1 & 75.00 & $11.95 \mathrm{~B}$ & $7.67 \mathrm{~B}$ & $0.90 \mathrm{~B}$ \\
\hline Peat :Sand 2:1 & 40.00 & $7.99 \mathrm{C}$ & $5.67 \mathrm{C}$ & $0.70 \mathrm{C}$ \\
\hline Peat :Sand 3:1 & 90.00 & $12.99 \mathrm{~A}$ & $9.67 \mathrm{~A}$ & $1.10 \mathrm{~A}$ \\
\hline
\end{tabular}

Means of agricultural media followed by the same letter within each column are not significantly different from each other at $1 \%$ level.

\section{REFERENCES}

1. Ahamed, D.M.R. 2009. Physiological studies on propagation of some indoor plants (In vitro).Thesis ,M.SC. Fac. of Agric. Moshtohor, Benha Univ.

2. Arafa, A.S. and I.A. Ibrahim. 1994. Effect of type of medium on growth and development of Cordyline and Gerbera plant culture in vitro. The $1^{\text {st }}$ Conf. of Ornamental Hort., 11:124-136.

3. Crane, E. (1990): Bees and beekeeping: Science, Practice and World Resources. Cornstock Publ., Ithaca, NY., USA. 593 pp.

4. Das, M.N. and N.C. Giri. 1986. Design and Analysis of Experiments, $2^{\text {nd } E d, ~}$ Published by Mohinder singh sejwal for Wiley, New Delhi 110002,488pp.

5. Duncan, D.B. (1955): Multiple range and multiple F-tests. Biometrices, 142.

6. Evaldsson, L.E. (1985): Shoot formation in vitro Cordyline terminalis. Hereditas suppl., 3:138. 
7. Evaldsson, L.E. and N.T. Welader. 1985. The effects of medium composition on in vitro propagation and in vivo growth of cordyline terminalis cv. Atoom. HortScience, 60:525-530.

8. Gunidy, F.L. 1990. Production of some fruit rootstocks through Tissue culture technique. Ph. D. Disert, Fac. Agric., Ain-Shams Univ., Cairo, Egypt.

9. Riordain, F.O.; P. Gallagher and I. Cormican. 1983. Micropropagation of Cordyline. Plant Tissue culture Relat Biatechnol., 105: 10.

10. MSTAT-C.(1990):Microcomputer statistical program for experiment design and analysis. MSTAT / Michigan State University, Michigan, USA.

11. Murashige, T. and F. Skoog. 1962. A revised medium for rapid growth and bioassays with tobacco tissue culture. Physiol. Plant, 15: 473-497.

12. Shahid J. B. (1999): In-vitro propagation of cabbage palm (Cordyline terminalis L.) through clonal regeneration. Anadolu, 1999, Vol.9, No.2, pp.56-59, 5 ref.

13. Tabio, C., J.D. Alvarez and M. Berisiartu. 1988. [Preliminary characterization of multifloral pollen from the El Cano area of Havana City Province, Cuba.]

14. Ciencia y Te'cnica en la Agricultura, Apicultura, 4: 73-81.

15. Welander, T. 1988. Micropropagation of Cordyline terminalis cv. Atoom. An European cooperation Project. Report Instition for Tradgardsvetenskap. Sveriges lantbruks Universities. No. 54. (Ornamental Hort, 15-724).

16. Youssef, Hanan. A.M. 2003. Induction of mutations and variations by using mutagens on some indoor plants. Ph.D. Thesis, Hort. Dept., Faclt. Of Agric., Moshtohor Univ. Zagazig, Egypt. 


\title{
أنتاج الكورادالين بأستخدام زراعة الانسجة
}

\author{
حنان محمد أحمد يوسف \\ قسم بحوث نباتات الزينة وتتسبق الحدائق - معهد بحوث البساتين - مركز البحوث الزراعية - الجيزة \\ - مهوربية مصر العربية
}

أجري هذا البحث بمعمل زراعه الأنسجة- معهد بحوث البساتين -مركز البحوث الزراعية

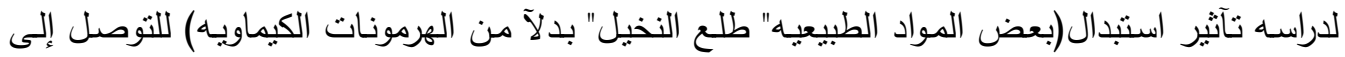

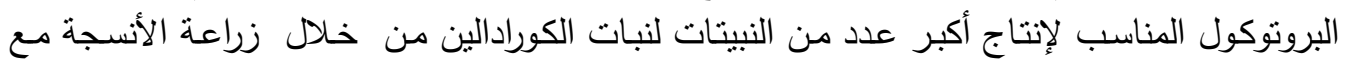
دراسة مراحل الزيادة العددية والتجذير والأقلمة خلال عامي 2009-2010. وأوضحت النير النتائج مايلى: لإنى

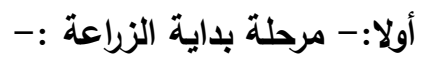
- نقع " القمة النامية " في محلول الكلوراكس بتركيز 20 \% ملمدة لمدة 20 دقيقة و العقلة ذات البرعم الواحد

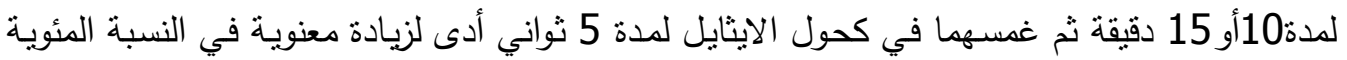
للنبيتات الحية وكذلك تقليل التلوث معنوياً .

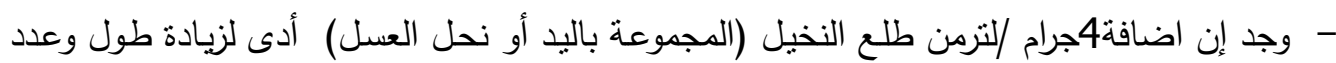

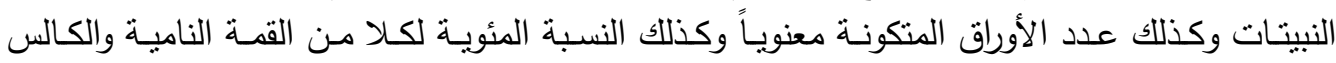

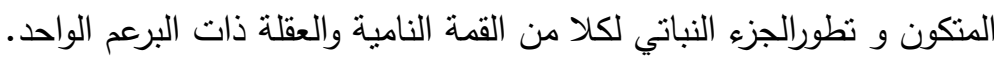

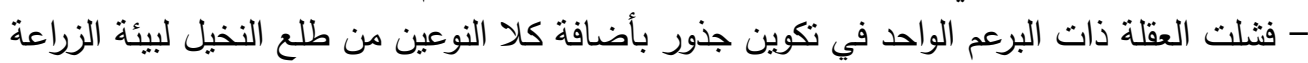

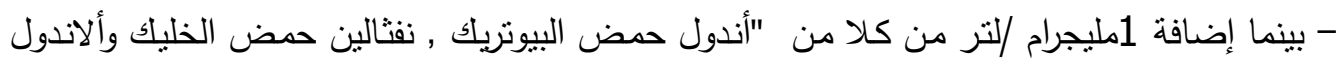

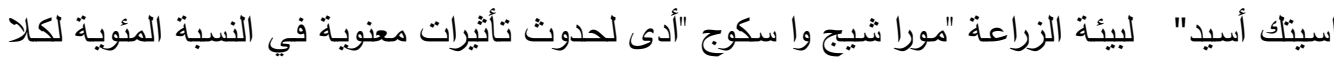

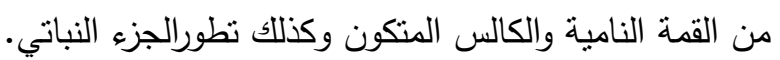

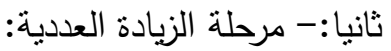

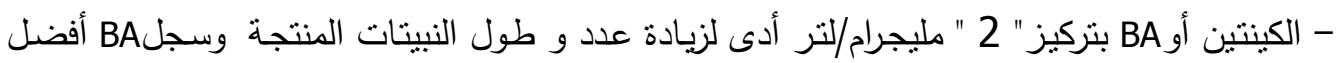
نتائج عن الكينتين. ثالثا:-مرحلة التجذير

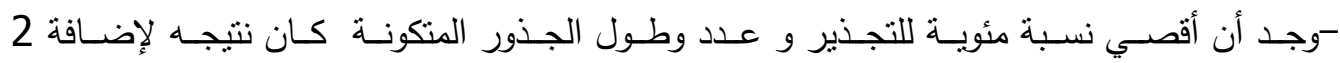

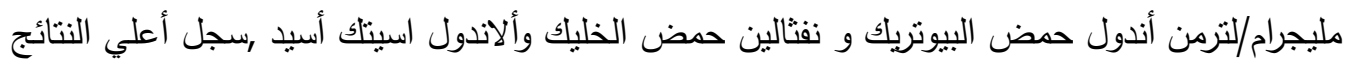

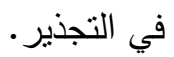

\section{رابعا:- مرحلة الآقلمة}

وجد أن زراعة النباتات فى بيئة مكونة من الرمل والبيت موس بنسبة 3:1 ذات ثأثثر إيجابى فى زيادة

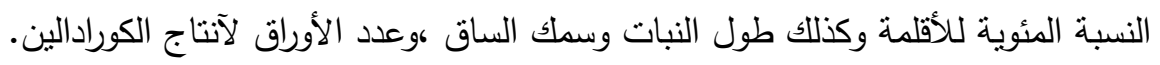
وعلي ذلك فأنة يتبين من النتائج السابقة مايلى لألى

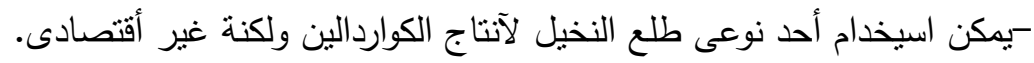

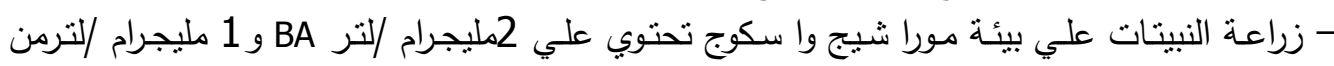

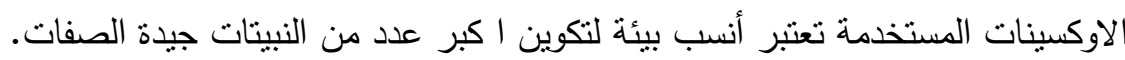
-زراعة النبيتات في التربة الزراعية "رمل وبيت موس بنسبة 3:1 كانئة كانت الأفضل. 